\title{
What else could create a change on our planet other than the gravity?
}

\begin{abstract}
Background: The Gravity is the force that attracts a body towards the center of the earth, and that's what we believe in. However the study worked on the fact and prove that no gravity is existing on the Earth.

Objectives: Analyze the impact in the high altitude how its increase toward the sea level and getting less in high mountain or sky.

Methods: The study included an experiment and Proofs based on and Theories and Sciences.

Conclusion: The study is proven the life with pressure is really important and living in high altitude could be dangerous if it exceed the normal range of the altitude. However, there is huge pressure on the earth that makes us breath, act and move with stability than the high altitude, where everything is floating.
\end{abstract}

Keywords: gravity, atmospheric pressure, temperature, hemoglobin, hyperbaric oxygen therapy
Volume 4 Issue 3 - 2017

\author{
Wael Awad Alshehri \\ Care National Hospital, Saudi Arabia
}

Correspondence: Wael Awad Alshehri, Care National Hospital, Riyadh, Saudi Arabia, Tel 0096658222700 I, Email Wael.alshehri@hotmail.com

Received: July 07, 2017 | Published: July 26, 2017

\section{Introduction}

\section{The gravity on the earth}

Long time ago we heard that the earth is running by gravity so it has a lot of control on everything on this life. But is it really the gravity that makes everything to be stable on this plant? I am actually disagreeing with that saying, because there is something that has greater effect than the gravity itself. It might be surprising, but in my opinion this is something worth to know and study it in details in order to help our world and too safe our life. Why I am claiming that? Because in my study, I found that the plant is controlled by the pressure more than the Gravity. Now in my study I am trying to proven the life with pressure is really important and living in high altitude could be dangerous if it exceed the normal range of the altitude. The amount of the pressure on the Earth is huge and makes everything's stable compare to the objects that located out of the atmosphere where is it floating.

\section{Methods}

When we talk about the pressure we find many things that affected by pressure. For example, the high altitude where the atmospheric pressure is lower than that at sea level. But when we are really close to the ground the percentage of the pressure is getting higher at the sea level. Also, there is a relationship with the atmospheric pressure and temperature. So, at the high altitude the weather is cool and the air wind is getting high, but when we are close to the ground the temperature is rising to the warmer side. This change in the pressure can also change many things on life of human, animal and the Nature as well. On my journey that I experience, I went to a place that is on the high altitude and I start to drive the car for about 2 hours from high altitude to see level (Figure 1).

As we know the atmospheric pressure at the high altitude has low pressure. So here is what happen, I had some of the water battle that is filled with half of a water. When I start getting close to the ground level the water battle is getting to shrink until it is half collapse. Then I return back from the low point at the ground that I have reached to the highest amount of the altitude and I notice that the water battle is back to the normal shape. For example in figure. 1 it explains the highest limit in the high altitude that I reached 2400 atmospheric pressure and going back to the see level which is around 650 atmospheric pressure. On the other hand, going from high altitude to the see level can effect on the human body, and more especially on the human ears.

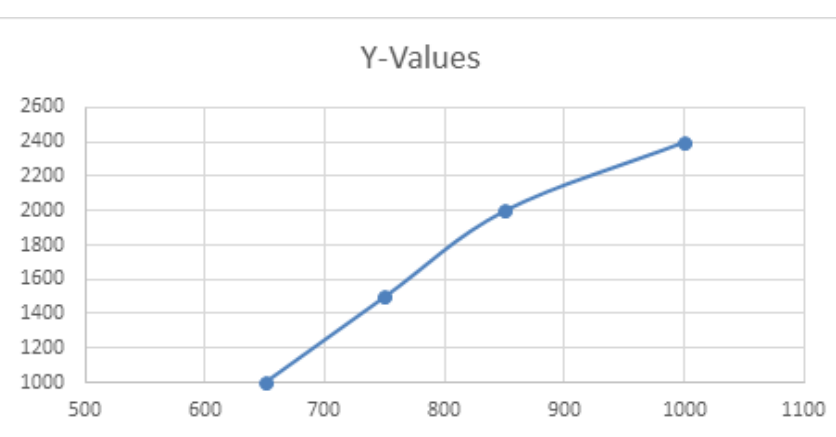

Figure I Illustrate the distance going from high level to low.

Explanation: These values explain how the distance that I expose to during the research. It started from high limit of the mounting which is around 2400 Meter, and going all the way to the ground near to 650 Meter of height. During the study, the atmospheric pressure is varying from the two distances which is less at the height and getting increase to the ground.

\section{Results}

The side effect on the human on the high altitude is really dangerous. "So as the altitude is getting higher and higher it increases the hemoglobin in the human blood. ${ }^{1}$ That because the human body consumes more oxygen. Also the partial pressure is decrease and that what make the persons hart rate, respiratory rate to be increasing and requires the need of oxygen supplement. So my question is how could 
the pressure make everything stable on the ground? When we talk about the gravity we are saying that there is something holding us on the earth of ground so we are stable with acceptable movement. But in realty there is huge pressure above us that forcing everything to be stable with flexible movement. Also when we need to move something from place to anther we need a strong power against the pressure to jamb or letting the airplane to fly in the sky and also a reversible power use to get down to sea level. So it is obviously that the gravity is not the one holding everything in the plant. I will clarify that the altitude is making an effect on our life especially when it comes to the living side. We cannot breathe very well in the high altitude and we might feel chest tightness. On the other hand we use in the medicine something called the Hyperbaric Oxygen Therapy. It is a therapeutic oxygen at pressures greater the $1 \mathrm{~atm}$. The pressure during the Hyperbaric Oxygen Therapy is expressed in atmospheric pressure absolute. So, one atmospheric pressure absolute is equals to $760 \mathrm{~mm} \mathrm{Hg}$ $(101.32 \mathrm{kPa})$. For example, in Figure 2 Hyperbaric Oxygen Therapy has physiologic effects on the body.

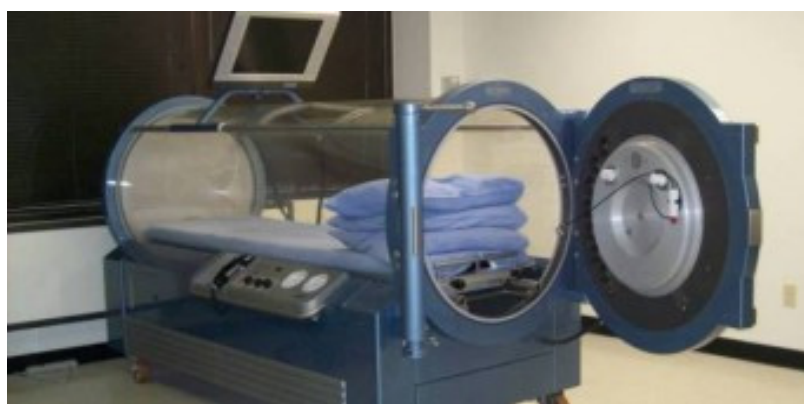

Figure 2 Hyperbaric oxygen therapy.

Illustrate about the hyperbaric therapy: The hyperbaric therapy is a device used in medicine to improve and process the inhalation. They use it by giving the patients $100 \%$ oxygen and they manipulate the atmospheric pressure inside the chamber. There is a lot of use Hyperbaric Oxygen Therapy, and they found out that it can help to enhanced host immune function, stroke, cerebral palsy, head injuries, and chronic fatigue have responded favorably to Hyperbaric Oxygen Therapy.

\section{Hyper oxygenation of blood and tissue}

Vasoconstriction, Enhanced host immune function, stroke, cerebral palsy, head injuries, and chronic fatigue have responded favorably to Hyperbaric Oxygen Therapy". ${ }^{2}$ Now in this study I will prove that the life with pressure is really important and living in high altitude is could be dangerous if it exceed the normal range of the altitude. Instead of that the earth might be running by pressure and there is less effect of the gravity. How to prove that? When the scientists goes to moon they must supply themselves with oxygen support because they are going to pass the normal altitude and no more oxygen at that stage.

Other example: When the airplane goes to the highest range of the altitude where the atmospheric pressure is low pressure, we can see obviously the people cannot control their movement as in the picture. In this case what is controlling the movement on the earth? Is the pressure have a great responsibility to control objects on the earth than the gravity? So I can say, the amount of the pressure on the earth is enough to control and stable the movement.

\section{Discussion}

Why all the objects is floating in the space, and when it comes to the earth it is more stable? It is very important question to let us to think what the idea behind that? Now I believe on the pressure more than the Gravity that control us on the earth. In compare to that, why the objects is not floating on the earth just like the space Figure 3. Sometimes when we go from high altitude to the see level, either by airplane or any other facility we feel headache and pain in the ears. It must prove that the gravity is not responsible about this at all. So in my opinion there is greater effect and control of the pressure and less of gravity.

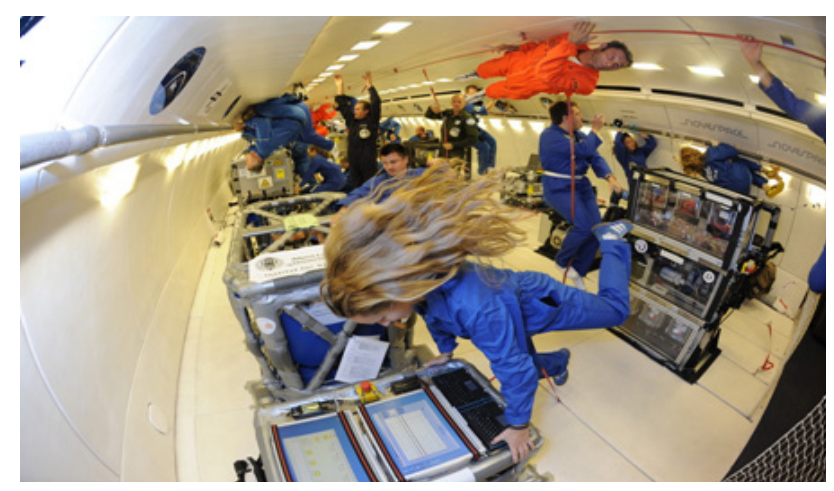

Figure 33 floating in the space.

Illustration: In the picture I want to prove that the more we go with the pressure the more we can find ourselves comfortable, stable with flexible movement and no need for extra support of oxygen. Also going outside the space can make the object to be floating and uncontrollable.

\section{Conclusion}

The research need more support and study in order to prove more fact about this idea. But in conclusion I found that the pressure in the high altitude makes the object to be free in floating and Lessing the percentage of $\mathrm{O}_{2}$ support. Also the movement from place to place is founded to be more difficult. On the other hand, when we go down to the sea level it is more controllable for the movement and stability. However, the more we go with the pressure the more we can find ourselves comfortable and no need for extra support of oxygen. It's actually enhance the oxygenation and make the persons berthing with comfortably. Where in the high altitude, it makes the persons feel chest tightness and difficulty of breathing. Furthermore, the fact behind writing this research is to prove that the earth is controlled by the pressure nor the Gravity. If we need to create a movement of any object we need force against the pressure just to move from place to another.

\section{Acknowledgements}

None.

\section{Conflicts of interest}

The authors declare that there is no conflict of interest.

\section{References}

1. San T, Polat S, Cingi C, et al. Effects of High Altitude on Sleep and Respiratory System and Theirs Adaptations. Scientific World Journal. 2013.

2. Latham E. Hyperbaric Oxygen Therapy. Overview, Hyperbaric Physics and Physiology, Contraindications. 2016. 